\title{
Microscopic analysis of multipole susceptibility of actinide dioxides: A scenario of multipole ordering in $\mathrm{AmO}_{2}$
}

\author{
Takashi Hotta \\ Department of Physics, Tokyo Metropolitan University, Hachioji, Tokyo 192-0397, Japan
}

(Dated: September 24, 2018)

\begin{abstract}
By evaluating multipole susceptibility of a seven-orbital impurity Anderson model with the use of a numerical renormalization group method, we discuss possible multipole states of actinide dioxides at low temperatures. In particular, here we point out a possible scenario for multipole ordering in americium dioxide. For $\mathrm{Am}^{4+}$ ion with five $5 f$ electrons, it is considered that the ground state is $\Gamma_{7}^{-}$ doublet and the first excited state is $\Gamma_{8}^{-}$quartet, but we remark that the $f^{5}$ ground state is easily converted due to the competition between spin-orbit coupling and Coulomb interactions. Then, we find that the $\Gamma_{8}^{-}$quartet can be the ground state of $\mathrm{AmO}_{2}$ even for the same crystalline electric field potential. In the case of $\Gamma_{8}^{-}$quartet ground state, the numerical results suggest that high-order multipoles such as quadrupole and octupole can be relevant to $\mathrm{AmO}_{2}$.

PACS numbers: 75.20.Hr, 75.40.Cx, 71.70.Ch, 71.27.+a
\end{abstract}

\section{INTRODUCTION}

Actinide dioxides with the fluorite structure of the space group $F m 3 m$ have been studied intensively for more than fifty years both from experimental and theoretical sides $: \frac{1,2}{}$ A typical target material is $\mathrm{UO}_{2}$ mainly due to its technological importance as a nuclear reactor fuel and a heterogeneous catalyst. As for theoretical research, a clear picture of the electronic structure in $\mathrm{UO}_{2}$ has been obtained. In fact, neutron scattering results show that $\mathrm{UO}_{2}$ is a noncollinear antiferromagnet below $30.8 \mathrm{~K}^{\underline{3}}$ Detailed analysis of core photoemission spectra has suggested that $\mathrm{UO}_{2}$ is an insulator of Mott-Hubbard type $\stackrel{\underline{4}}{\underline{\underline{*}}}$ The crystalline electric field $(\mathrm{CEF})$ states of $\mathrm{UO}_{2}$ have been also determined $\underline{\underline{5}}$

Concerning $\mathrm{NpO}_{2}$, over fifty years since $1953, \underline{6}$ it has been known to exhibit a mysterious low-temperature ordered phase $\stackrel{7,8}{ }$ Several phenomenological works on the ordered phase have claimed a key role of octupole degree of freedom $9.10,11,12,13$ The CEF states of $\mathrm{NpO}_{2}$ have been determined by neutron scattering experiment. 14 Recently, the octupole order has been strongly supported by ${ }^{17} \mathrm{O}-\mathrm{NMR}$ experiment ${ }^{15}$ and by inelastic neutron scattering study 16 In order to understand why such high-order multipole ordering appears, it is necessary to proceed to the research in a microscopic level. For this issue, it has been shown that octupole order actually characterizes the ground state of $\mathrm{NpO}_{2}$ by the analysis of an $f$-electron model on the basis of a $j$ - $j$ coupling scheme on an fcc lattice,$\frac{17}{18}$

On the other hand, $\mathrm{PuO}_{2}$ is known to be a semiconductor with magnetic susceptibility which is almost independent of temperature up to $1000 \mathrm{~K}$, since the CEF ground state is $\Gamma_{1}^{+}$singlet and the first excited state is $\Gamma_{4}^{+}$ triplet with the large excitation energy as $123 \mathrm{meV}: \underline{\underline{19,20}}$ Thus, from the viewpoint of magnetism, $\mathrm{PuO}_{2}$ did not attract much attention.

Now let us turn our attention to $\mathrm{AmO}_{2}$. Probably due to the difficulty in the treatment of this material with high radioactivity, we cannot find lots of experimental results on $\mathrm{AmO}_{2}$. In 1969, Mössbauer isomer shift in $\mathrm{AmO}_{2}$ was measured $\stackrel{21}{2}$ After that, the magnetic susceptibility was measured and the peak was found around at $15 \mathrm{~K} \stackrel{22}{2}$ Naively thinking, such a peak seems to suggest the signal of antiferromagnetic ordering, while neutron diffraction measurement did not detect antiferromagnetic order in agreement with the Mössbauer measurement ${ }^{23}$ This situation looks similar to that of $\mathrm{NpO}_{2}$. Namely, multipole degree of freedom seems to be a key issue in $\mathrm{AmO}_{2}$ to reconcile experimental results, as has been proposed for $\mathrm{NpO}_{2}$ in the context of octupole ordering.

Here we note that the CEF ground state of $\mathrm{AmO}_{2}$ was considered to be $\Gamma_{7}^{-}$doublet from the experimen-

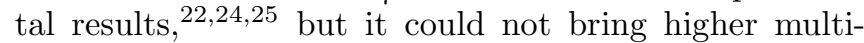
poles. This is in sharp contrast to the case of $\mathrm{NpO}_{2}$ with the confirmed CEF ground state of $\Gamma_{8}^{-}$quartet. Due to the CEF analysis of actinide dioxides, the $\Gamma_{7}^{-}$doublet ground state has been suggested $\stackrel{26}{r}$ Thus, it seems to be the mainstream in the research of actinide dioxides to clarify a mechanism which explains the disappearance of antiferromagnetic order for the $\Gamma_{7}^{-}$doublet ground state.

However, we believe that there still exists an alternative scenario on the basis of multipole ordering in $\mathrm{AmO}_{2}$, when we recall the fact that the CEF ground state of the $f^{5}$ electron system is easily converted due to the competition between spin-orbit coupling and Coulomb interaction 27 This point has been also discussed by the present author to propose a possible scenario which explains the change of the CEF ground state among Sm-based filled skutterudite compounds, $\stackrel{28}{\underline{ }}$ since trivalent $\mathrm{Sm}$ ion includes five $4 f$ electrons. It has been experimentally $29,30,31,32$ and theoretically $29,30,31,32,33$ shown that the angular momentum coupling of the $5 f$ states of Am is situated between the $L S$ and $j$-j coupling limits for many chemical situations, albeit closer to the $j$ - $j$ limit. Thus, a strong spin-orbit coupling is present in the $5 f$ states of $f^{5}$ and $f^{6}$ configurations of Am that can compete with Coulomb interactions. Accordingly, it is believed to be meaningful to pursue a possibility of multipole ordering in $\mathrm{AmO}_{2}$ 
with the $\Gamma_{8}^{-}$quartet ground state.

In this paper, we show that multipole order is possible in $\mathrm{AmO}_{2}$, when we appropriately take into account both spin-orbit coupling and Coulomb interaction in the $f$-electron terms for the CEF ground state. In the case of five $f$ electrons such as $\mathrm{Am}^{4+}$ ion, the ground state is easily converted between $\Gamma_{7}^{-}$and $\Gamma_{8}^{-}$, when spin-orbit coupling and Coulomb interaction compete with each other. Then, the $\Gamma_{8}^{-}$quartet can be the ground state, even if the CEF potential is unchanged. In order to see what type of multipole is relevant, we evaluate the multipole susceptibility of the Anderson model by using a numerical renormalization group method. We find that higherorder multipoles are actually relevant to $\mathrm{AmO}_{2}$ within the present calculation.

The organization of this paper is as follows. In Sec. II, we discuss the local $f$-electron state emerging from the competition among the Coulomb interaction and spinorbit coupling under the CEF potential. In particular, the change of the $f^{5}$ ground state is explained in detail. In Sec. III, we show the model Hamiltonian. In order to discuss multipole properties, it is necessary to define the multipole operator. Here we explain the description of the multipole as spin-charge complex one electron operator. Then, we briefly explain the numerical technique used in this paper. In Sec. IV, we show the results of the multipole state for the case of $n=2 \sim 5$, where $n$ is the local $f$-electron number. In particular, the results for $n=5$ are discussed in detail. Finally, the paper is summarized in Sec. V. Throughout this paper, we use such units as $\hbar=k_{\mathrm{B}}=1$.

\section{LOCAL $f$-ELECTRON STATE}

Let us first discuss the local $f$-electron states of actinide ions. The local Hamiltonian should be composed of three parts as

$$
H_{\mathrm{loc}}=H_{\mathrm{so}}+H_{\mathrm{int}}+H_{\mathrm{CEF}} .
$$

The first term denotes the spin-orbit coupling, given by

$$
H_{\mathrm{so}}=\lambda \sum_{m, m^{\prime}} \sum_{\sigma, \sigma^{\prime}} \zeta_{m, \sigma, m^{\prime}, \sigma^{\prime}} f_{m \sigma}^{\dagger} f_{m^{\prime} \sigma^{\prime}},
$$

where $\sigma=+1(-1)$ for up (down) spin, $f_{m \sigma}$ is the annihilation operator for $f$ electron with spin $\sigma$ and $z$ component $m$ of angular momentum $\ell=3, \lambda$ is the spinorbit coupling, $\zeta_{m, \pm 1, m, \pm 1}= \pm m / 2, \zeta_{m \pm 1, \mp 1, m, \pm 1}=$ $\sqrt{12-m(m \pm 1)} / 2$, and zero for the other cases.

The second term indicates the Coulomb interaction among $f$ electrons, expressed as

$$
H_{\mathrm{int}}=\sum_{m_{1} \sim m_{4}} \sum_{\sigma, \sigma^{\prime}} I_{m_{1} m_{2}, m_{3} m_{4}} f_{m_{1} \sigma}^{\dagger} f_{m_{2} \sigma^{\prime}}^{\dagger} f_{m_{3} \sigma^{\prime}} f_{m_{4} \sigma}
$$

where the Coulomb integral $I_{m_{1}, m_{2}, m_{3}, m_{4}}$ is given by

$$
I_{m_{1}, m_{2}, m_{3}, m_{4}}=\sum_{k=0}^{6} F^{k} c_{k}\left(m_{1}, m_{4}\right) c_{k}\left(m_{2}, m_{3}\right) .
$$

Here $F^{k}$ is the radial integral for the $k$-th partial wave, called Slater integral or Slater-Condon parameter ${ }^{34,35}$ and $c_{k}$ is the Gaunt coefficient. 36.37 Note that the sum is limited by the Wigner-Eckart theorem to even values $(k=0,2,4$, and 6$)$.

The third term is the CEF potential, given in the oneelectron potential form as

$$
H_{\mathrm{CEF}}=\sum_{m, m^{\prime}} \sum_{\sigma} B_{m, m^{\prime}} f_{m \sigma}^{\dagger} f_{m^{\prime} \sigma}
$$

where $B_{m, m^{\prime}}$ is the CEF potential. Since the fluorite structure belongs to $O_{\mathrm{h}}$ point group, $B_{m, m^{\prime}}$ is given by using a couple of CEF parameters $B_{4}^{0}$ and $B_{6}^{0}$ for angular momentum $\ell=3$ as $^{38}$

$$
\begin{aligned}
& B_{3,3}=B_{-3,-3}=180 B_{4}^{0}+180 B_{6}^{0} \\
& B_{2,2}=B_{-2,-2}=-420 B_{4}^{0}-1080 B_{6}^{0} \\
& B_{1,1}=B_{-1,-1}=60 B_{4}^{0}+2700 B_{6}^{0} \\
& B_{0,0}=360 B_{4}^{0}-3600 B_{6}^{0} \\
& B_{3,-1}=B_{-3,1}=60 \sqrt{15}\left(B_{4}^{0}-21 B_{6}^{0}\right) \\
& B_{2,-2}=300 B_{4}^{0}+7560 B_{6}^{0} .
\end{aligned}
$$

Note the relation of $B_{m, m^{\prime}}=B_{m^{\prime}, m}$. Following the traditional notation, $\stackrel{39}{,}$ we define

$$
B_{4}^{0}=W x / F(4), B_{6}^{0}=W(1-|x|) / F(6),
$$

where $x$ and the sign of $W$ specify the CEF energy scheme, while $|W|$ determines the energy scale for the CEF potential. Concerning non-dimensional parameters, $F(4)$ and $F(6)$, we use $F(4)=15$ and $F(6)=180$ for $\ell=3.38$

Here we briefly explain the parameters of the local Hamiltonian. Concerning Slater-Condon parameters, first we set $F^{0}=10 \mathrm{eV}$ by hand, since we are not interested in the determination of the absolute value of the ground state energy. Others are determined so as to reproduce excitation spectra of $\mathrm{U}^{4+}$ ion with two $5 f$ electrons. 40 Here we show only the results: $F^{2}=6.36 \mathrm{eV}, F^{4}=5.63$ $\mathrm{eV}$, and $F^{6}=4.13 \mathrm{eV}$. As for spin-orbit coupling $\lambda$, we use the values of actinide atoms such as $\lambda=0.235 \mathrm{eV}(\mathrm{U})$, $0.272 \mathrm{eV}(\mathrm{Np}), 0.311 \mathrm{eV}(\mathrm{Pu})$, and $0.351 \mathrm{eV}(\mathrm{Am})$.

For the estimation of the CEF parameters, let us summarize the CEF energy levels of actinide dioxides. For $\mathrm{UO}_{2}$, the ground state is $\Gamma_{5}^{+}$triplet and the first excited state is $\Gamma_{3}^{+}$doublet with the excitation energy $150 \mathrm{meV} . \underline{\underline{5}}$ For $\mathrm{NpO}_{2}$, the ground and first excited states are, respectively, $\Gamma_{8}^{-(2)}$ and $\Gamma_{8}^{-(1)}$ quartets with the excitation energy $55 \mathrm{meV} .{ }^{14}$ For $\mathrm{PuO}_{2}$, the ground state is $\Gamma_{1}^{+}$singlet, while the first excited state is $\Gamma_{4}^{+}$triplet with the excitation energy $123 \mathrm{meV}, 19,20$

Now we set the CEF parameters for actinide dioxides in the present notation. For the purpose, first we estimate $W$ and $x$ so as to reproduce the CEF scheme of $\mathrm{UO}_{2}$ by the $f^{2}$ electrons state. This is not a difficult task, since the CEF parameters are easily restricted from the experimental results. After that, among the values of $W$ and $x$ appropriate for $\mathrm{UO}_{2}$, we further restrict the values of $W$ and $x$ which can also reproduce the results 


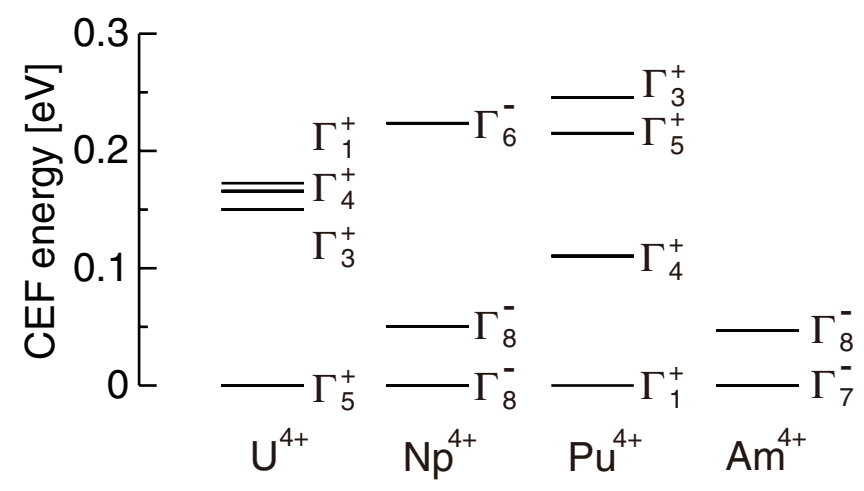

FIG. 1: CEF energy level schemes for tetravalent actinide ions. Parameters used here are explained in the maintext.

for $\mathrm{NpO}_{2}$ and $\mathrm{PuO}_{2}$. Note that the CEF states for $f^{3}$ and $f^{4}$ states are almost reproduced by using the parameters of $f^{2}$ electron state. Since the CEF term is just given by the one-electron potential, the CEF effect is not so drastically changed among the materials with the same crystal structure, even though the $f$-electron number is different.

After some calculations, we determine $W=-10.5 \mathrm{meV}$ and $x=0.62$. The results for the CEF level schemes are summarized in Fig. 1. It is observed that the CEF states of $\mathrm{UO}_{2}, \mathrm{NpO}_{2}$, and $\mathrm{PuO}_{2}$ are well reproduced. Note here that for the same CEF parameters, the ground state for $\mathrm{Am}^{4+}$ is found to be $\Gamma_{7}^{-}$doublet and the excited state is $\Gamma_{8}^{-}$quartet with the excitation energy of about $50 \mathrm{meV}$. This is consistent with the previous theoretical results on the CEF states of $\mathrm{AmO}_{2}$ obtained by more detailed calculations. 26

Naively thinking, there occurs ordering of magnetic moment originating from $\Gamma_{7}^{-}$ground state for $\mathrm{AmO}_{2}$, but it seems to be contradict with neutron diffraction study. In order to resolve such contradiction, there are two ways: One is to consider a mechanism which explains the disappearance of magnetic moment even in the $\Gamma_{7}^{-}$ground state. Another way is to reconsider the local $f$-electron term by focusing on spin-orbit coupling and Coulomb interaction. Here we propose an alternative scenario on the basis of the second direction.

Thus far, we have simply assumed that Coulomb interaction is not changed among different actinide ions, but in actuality, they may be changed. Concerning spin-orbit coupling, we have used the values in actinide atom, but it may be also changed in the ionic states. Then, we point out that for $f^{5}$ systems, the CEF states are sensitively changed by the competition between Coulomb interaction and spin-orbit coupling in comparison with other values of local $f$-electron number 27,28

In order to understand that the effect of Coulomb interaction and spin-orbit coupling appears in the CEF parameters, here we express the CEF parameter $B_{4}^{0}$ by using the so-called Stevens factor as

$$
B_{4}^{0}=A_{4}\left\langle r^{4}\right\rangle \beta_{J}^{(n)},
$$

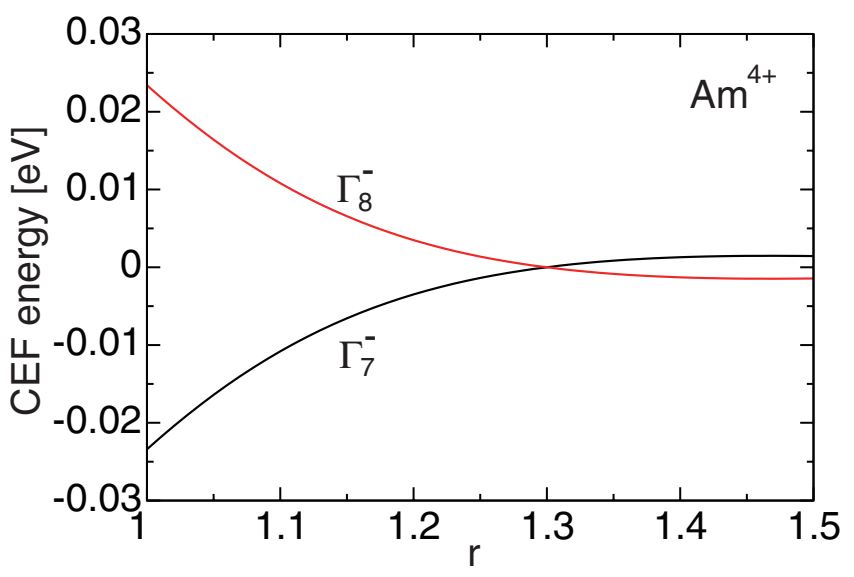

FIG. 2: The CEF energy for $n=5$ vs. $r$. The meaning of $r$ is explained in the maintext.

where $A_{k}$ is the parameter depending on materials, $\left\langle r^{k}\right\rangle$ denotes the radial average of local $f$-electron wavefunction, $n$ denotes the local $f$-electron number, $J$ is the total angular momentum of the ground state multiplet, and $\beta_{J}^{(n)}$ indicates the Stevens factor, which is one of coefficients appearing in the method of Stevens' operator equivalent $\underline{\underline{41}}$

For the case of $n=5$, it is well known that the ground state multiplet is characterized by $J=5 / 2$. After lengthy calculations, for $n=5$ and $J=5 / 2$, we can obtain that $\beta_{5 / 2}^{(5)}=(13 / 21) \beta_{3}$ in the $L S$ coupling scheme and $\beta_{5 / 2}^{(5)}=-(11 / 7) \beta_{3}$ in the $j-j$ coupling scheme,,$\underset{27}{\underline{n}}$ where $\beta_{3}$ denotes the Stevens factor for $\ell=3$, given by $\beta_{3}=2 / 495$. It should be noted that the sign of $\beta_{J}^{(n)}$ is changed between the $L S$ and $j$ - $j$ coupling schemes, suggesting that the ground state is converted, when Coulomb interaction and/or spin-orbit coupling are changed.

The modification of Coulomb interaction and spinorbit coupling is closely related to the picture for multi$f$-electron state. The CEF level schemes in Fig. 1 is qualitatively understood by a $j-j$ coupling scheme. Namely, by assuming that the effective Hund's rule coupling is smaller than the CEF level splitting, we simply accommodate plural numbers of $f$ electrons in the levels of $\Gamma_{8}^{-}$ground and $\Gamma_{7}^{-}$excited states. Then, we can easily reproduce all the $\mathrm{CEF}$ level schemes of tetravalent actinide ions. However, the conversion of the CEF ground state for $n=5$ indicates that the actual situation should be slightly shifted to the side of the $L S$ coupling scheme. We note that due to such a shift, the CEF states for $n=2$, 3 , and 4 are not qualitatively changed, while in the case of $n=5$, the ground state is converted.

Here we emphasize that such a ground-state conversion occurs in the region of realistic values of Coulomb interaction and spin-orbit coupling, as has been pointed out in the discussion of the CEF states of Sm-based filled skutterudites $\stackrel{28}{2}$ In fact, as mentioned in Sec. I, it has been shown that the angular momentum coupling of the 
$5 f$ states of Am is situated between the $L S$ and $j-j$ coupling limits, but it is rather closer to the $j-j$ coupling limit 29,30,31,32,33 Thus, it seems to be reasonable to change slightly spin-orbit coupling and/or Coulomb interaction in the case of tetravalent Am ion.

If we resort to first-principles calculation, we may determine correctly $F^{j}$ and $\lambda$, but it is out of the scope of the present paper. Here we simply introduce an artificial parameter $r$ to control Coulomb interactions and spinorbit coupling as $F^{k} \rightarrow r F^{k}$ and $\lambda \rightarrow \lambda / r$ with $r \geq 1$.

In Fig. 2, we depict the energies of $H_{\text {loc }}$ as functions of $r$ for $n=5$. We find that the conversion of the ground state occurs around at $r \approx 1.3$. Note that in other values of $n$, the ground states are not changed. Thus, we can obtain the $\Gamma_{8}^{-}$quartet ground state for $\mathrm{Am}^{4+}$. In the following sections, we will discuss how the multipole states actually appear when we change the value of $r$ for Am ion.

\section{MODEL AND METHOD}

\section{A. Anderson model}

Now we include the hybridization between localized and conduction electrons. The Hamiltonian is the Anderson model, given by

$$
H=\sum_{\mathbf{k}, \sigma} \varepsilon_{\mathbf{k}} c_{\mathbf{k} \sigma}^{\dagger} c_{\mathbf{k} \sigma}+\sum_{\mathbf{k}, \sigma, m}\left(V_{m} c_{\mathbf{k} \sigma}^{\dagger} f_{m \sigma}+\text { h.c. }\right)+H_{\mathrm{loc}},
$$

where $\varepsilon_{\mathbf{k}}$ denotes conduction electron dispersion, $c_{\mathbf{k} \sigma}$ indicates the annihilation operator for conduction electron with momentum $\mathbf{k}$ and spin $\sigma$, and $V_{m}$ is the hybridization between conduction and $f$ electrons.

Note that we consider only $a_{\mathrm{u}}$ single conduction band with xyz symmetry composed of oxygen $2 p$ electrons. Since oxygen ions surrounding actinide ions are located in the $[1,1,1]$ direction, there should exist a conduction band composed of $2 p$ electrons with xyz symmetry. This picture seems to be consistent with band-structure calculation, ${ }^{42}$ but the ignorance of $t_{1 \mathrm{u}}$ and $t_{2 \mathrm{u}}$ bands is just assumption. Here we note that the hybridization occurs between the states with the same symmetry of local $f$-electron state. Since the $a_{\mathrm{u}}$ conduction band has xyz symmetry, we set $V_{2}=-V_{-2}=V$ and zero for other $m$. Hereafter, a half of the bandwidth of $a_{\mathrm{u}}$ conduction band $D$ is set as the energy unit, i.e., $D=1 \mathrm{eV}$. We fix $V$ as $V / D=0.05$ throughout this paper. Note that in order to adjust the local $f$-electron number $n$, we appropriately change the chemical potential in the actual calculation, although we do not explicitly show such a term.

\section{B. Multipole operator}

In order to discuss multipole properties, it is necessary to define the multipole operator $\hat{X}$ for $f$ electron $43,44,45,46,47,48$ In general, $\hat{X}$ is expressed as

$$
\hat{X}=\sum_{k, \gamma} p_{\gamma}^{(k)} \hat{T}_{\gamma}^{(k)}
$$

where $k$ is a rank of multipole, $\gamma$ is a label to express $O_{\mathrm{h}}$ irreducible representation, and $\hat{T}_{\gamma}^{(k)}$ is cubic tensor operator, given by $\hat{T}_{\gamma}^{(k)}=\sum_{q} G_{\gamma, q}^{(k)} \hat{T}_{q}^{(k)}$. Here an integer $q$ runs between $-k$ and $k, \hat{T}_{q}^{(k)}$ is spherical tensor operator, and $G_{\gamma, q}^{(k)}$ is the transformation matrix between spherical and cubic harmonics. We determine $p_{\gamma}^{(k)}$ later.

In order to obtain explicit expression of the spherical tensor operator $\hat{T}_{q}^{(k)}$, it is convenient to convert the $f$-electron basis from $(m, \sigma)$ to $(j, \mu)$, where $j$ is the total angular momentum and $\mu$ is the $z$-component of $j$. When we define $f_{j \mu}$ as the annihilation operator for $f$ electron labeled by $j$ and $\mu$, we obtain $\hat{T}_{q}^{(k)}$ in the secondquantized form as

$$
\hat{T}_{q}^{(k)}=\sum_{j, \mu, \mu^{\prime}} T_{j ; \mu, \mu^{\prime}}^{(k, q)} f_{j \mu}^{\dagger} f_{j \mu^{\prime}}
$$

Note that there are no components between different values of $j$, since the matrix for total angular momentum is block-diagonalized in the basis of $(j, \mu)$. The matrix element of $T_{j ; \mu, \mu^{\prime}}^{(k, q)}$ is calculated by the Wigner-Eckart theorem as

$$
T_{j ; \mu, \mu^{\prime}}^{(k, q)}=\frac{\left\langle j\left\|T^{(k)}\right\| j\right\rangle}{\sqrt{2 j+1}}\left\langle j \mu \mid j \mu^{\prime} k q\right\rangle
$$

where $\left\langle j \mu \mid j \mu^{\prime} k q\right\rangle$ indicates the Clebsch-Gordan coefficient and $\left\langle j\left\|T^{(k)}\right\| j\right\rangle$ denotes the reduced matrix element for spherical tensor operator, given by $\left\langle j\left\|T^{(k)}\right\| j\right\rangle=$ $\sqrt{(2 j+k+1) ! /(2 j-k) !} / 2^{k}$. Note that $k \leq 2 j$ and the highest rank of $f$-electron multipole is 7 .

Let us now determine the coefficient $p_{\gamma}^{(k)}$. In order to discuss the multipole state, it is necessary to evaluate the multipole susceptibility in the linear response theory. However, multipoles belonging to the same symmetry are mixed in general, even if the rank is different. In addition, multipoles are also mixed due to the CEF effect. Thus, we determine $p_{\gamma}^{(k)}$ by the normalized eigenstate of susceptibility matrix

$$
\begin{aligned}
\chi_{k \gamma, k^{\prime} \gamma^{\prime}} & =\frac{1}{Z} \sum_{i, j} \frac{e^{-E_{i} / T}-e^{-E_{j} / T}}{E_{j}-E_{i}}\left\langle i\left|\left[\hat{T}_{\gamma}^{(k)}-\rho_{\gamma}^{(k)}\right]\right| j\right\rangle \\
& \times\left\langle j\left|\left[\hat{T}_{\gamma^{\prime}}^{\left(k^{\prime}\right)}-\rho_{\gamma^{\prime}}^{\left(k^{\prime}\right)}\right]\right| i\right\rangle,
\end{aligned}
$$

where $E_{i}$ is the eigenenergy for the $i$-th eigenstate $|i\rangle$ of $H, T$ is a temperature, $\rho_{\gamma}^{(k)}=\sum_{i} e^{-E_{i} / T}\left\langle i\left|\hat{T}_{\gamma}^{(k)}\right| i\right\rangle / Z$, and $Z$ is the partition function given by $Z=\sum_{i} e^{-E_{i} / T}$. Note that the multipole susceptibility is given by the eigenvalue of the susceptibility matrix. 


\section{Method}

In order to evaluate multipole susceptibility of the Anderson model, we employ a numerical renormalization group (NRG) method. ${ }^{49}$ in which momentum space is logarithmically discretized to include efficiently the conduction electrons near the Fermi energy and the conduction electron states are characterized by "shell" labeled by $N$. The shell of $N=0$ denotes an impurity site described by the local Hamiltonian.

In the NRG method, we transform the Hamiltonian into the recursion form as

$$
H_{N+1}=\sqrt{\Lambda} H_{N}+t_{N} \sum_{\sigma}\left(c_{N \sigma}^{\dagger} c_{N+1 \sigma}+c_{N+1 \sigma}^{\dagger} c_{N \sigma}\right)
$$

where $\Lambda$ denotes a parameter for logarithmic discretization, $c_{N \sigma}$ indicates the annihilation operator of conduction electron in the $N$-shell, and $t_{N}$ is the hopping of electron between $N$ - and $(N+1)$-shells, expressed by

$$
t_{N}=\frac{\left(1+\Lambda^{-1}\right)\left(1-\Lambda^{-N-1}\right)}{2 \sqrt{\left(1-\Lambda^{-2 N-1}\right)\left(1-\Lambda^{-2 N-3}\right)}} .
$$

The initial term $H_{0}$ is given by

$$
H_{0}=\Lambda^{-1 / 2}\left[H_{\mathrm{loc}}+\sum_{\sigma} V\left(c_{0 \sigma}^{\dagger} f_{\mathrm{c} \sigma}+f_{\mathrm{c} \sigma}^{\dagger} c_{0 \sigma}\right)\right] .
$$

Each component of multipole susceptibility matrix eq. (13) is evaluated by using the renormalized state. Then, the multipole state is defined by the eigen states of eq. (13). We note that the temperature $T$ is defined as $T=\Lambda^{-(N-1) / 2}$ in the NRG calculation, where $N$ is the number of the renormalization step. Due to the limitation of computer resources, we keep only $M$ low-energy states. In this paper, we set $\Lambda=5$ and $M=3000$.

\section{RESULTS}

Now we discuss the multipole state of the Anderson model eq. (9). In Figs. 3(a)-(c), we show $T \chi_{\gamma}$ vs. $T$ for $n=2,3$, and 4 , where $\chi_{\gamma}$ is the eigenvalue of the multipole susceptibility. We use the same values of the parameters in the Hamiltonian for $n=2,3$, and 4 . The values of the spin-orbit coupling are $\lambda=0.235 \mathrm{eV}, 0.272 \mathrm{eV}$, and $0.311 \mathrm{eV}$ for $n=2,3$, and 4 , respectively. The eigenstates are classified by irreducible representation of $O_{\mathrm{h}}$ point group. Here we use short-hand notations such as " $3 \mathrm{~g}$ " and " $5 u$ ", which denote $\Gamma_{3}^{+}$and $\Gamma_{5}^{-}$, respectively, in the Bethe notation. Note that " $1 \mathrm{u}$ " does not appear among multipoles up to rank 7 .

For $n=2\left(\mathrm{UO}_{2}\right)$, we find the optimized state is labeled by $4 \mathrm{u}$, which is mainly composed of dipole (about $92 \%)$. The secondary components are quadrupoles $(5 \mathrm{~g}$ and $3 \mathrm{~g})$, but $\chi_{5 \mathrm{~g}}$ and $\chi_{3 \mathrm{~g}}$ is smaller in one order in comparison with $\chi_{4 \mathrm{u}}$. For $n=3\left(\mathrm{NpO}_{2}\right)$, as easily deduced from the $\Gamma_{8}^{-}$quartet ground state, we find varieties of
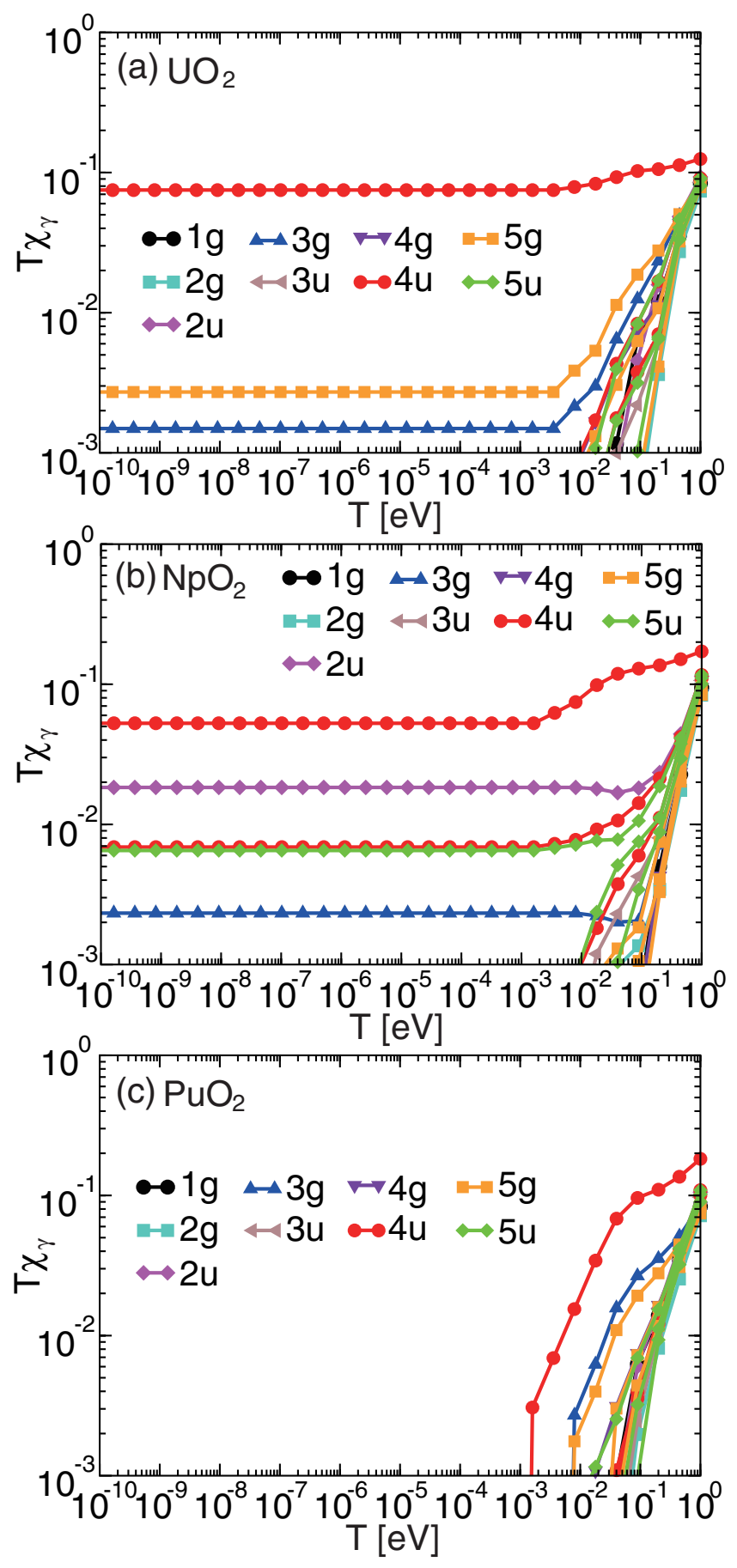

FIG. 3: $T \chi_{\gamma}$ vs. temperature $T$ for (a) $\mathrm{UO}_{2}$, (b) $\mathrm{NpO}_{2}$, and (c) $\mathrm{PuO}_{2}$.

multipoles. Among them, the primary component is $4 \mathrm{u}$, which is mainly composed of dipole (about 96\%). The secondary one is $2 \mathrm{u}$ octupole. In the third group, another $4 \mathrm{u}$ and $5 \mathrm{u}$ are almost degenerate. We note that this $4 \mathrm{u}$ is composed of higher multipole component and the $5 \mathrm{u}$ is mainly composed of octupole. The fourth component is $3 \mathrm{~g}$ quadrupole. We emphasize the existence of octupoles $(2 \mathrm{u}$ and $5 \mathrm{u})$ with significant eigenvalues. 

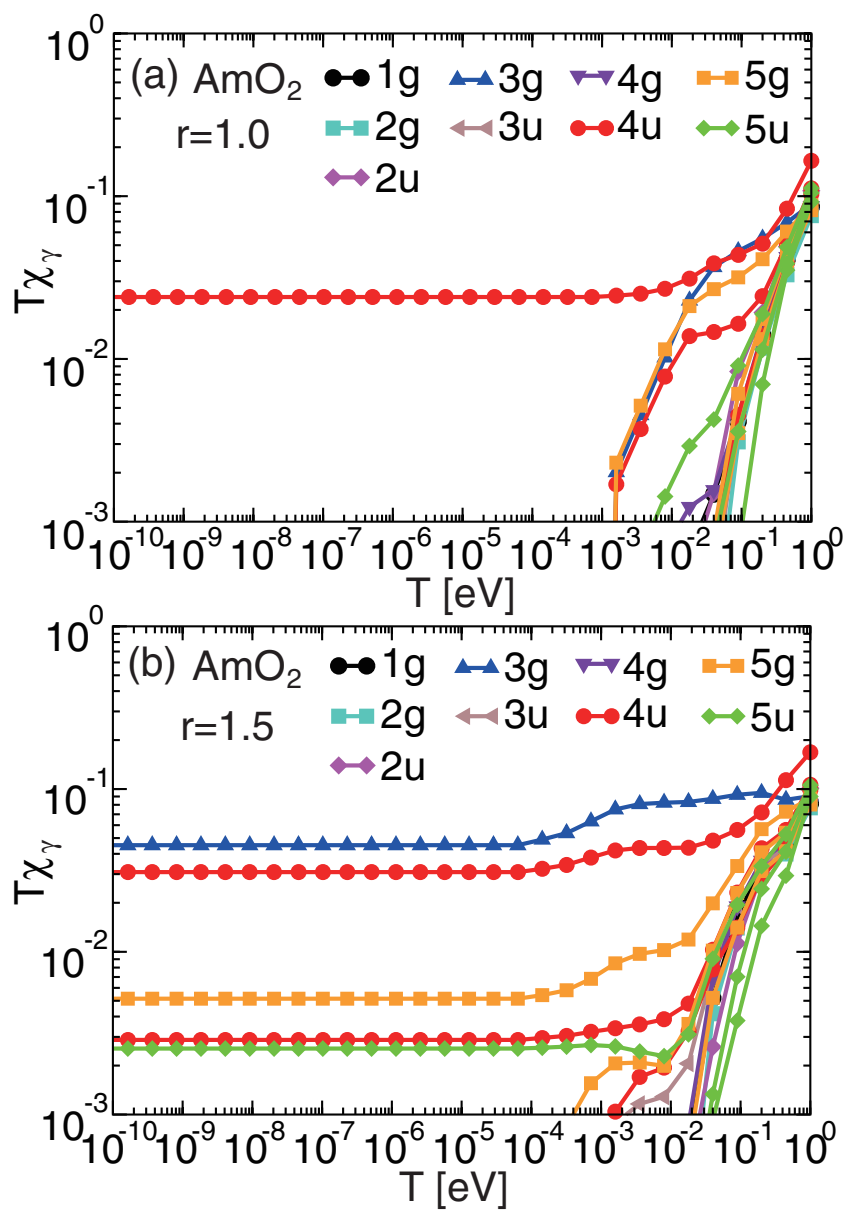

FIG. 4: (a) $T \chi_{\gamma}$ vs. temperature $T$ for $n=5$. The parameters are the same as those in Fig. 2, except for the spin-orbit coupling. (b) $T \chi_{\gamma}$ vs. temperature $T$ for $n=5$ and $r=1.5$ with enhanced Coulomb interaction and reduced spin-orbit coupling.

In the present calculation, we cannot determine the kind of multipole ordering in actual systems. However, the multipoles which remain at low temperatures are the candidates which will order in the actual system. In the case of $\mathrm{NpO}_{2}$, it has been gradually revealed that triple-q order of $5 \mathrm{u}$ octupole can naturally reconcile several kinds of experiments. The $5 \mathrm{u}$ octupole is actually included in the multipoles in the present calculations, although it is not dominant component.

For $n=4\left(\mathrm{PuO}_{2}\right)$, we do not find any significant multipole component, as easily understood from the $\Gamma_{1}^{+}$singlet ground state which is well separated from the magnetic excited state. In this sense, from the viewpoint of magnetism, this case does not attract much attention.

Next we move on to the case of $n=5$, corresponding to $\mathrm{AmO}_{2}$. In Fig. 4(a), we show the results for $n=5$ by using the parameters for $r=1$. Namely, the Coulomb interactions are the same as those in Figs. 3(a)-(c). The spin-orbit coupling is set as $\lambda=0.351 \mathrm{eV}$, which is the value for Am atom. In this case, since the ground state is $\Gamma_{7}^{-}$doublet, the component which remain in the lowtemperature region is $4 \mathrm{u}$, which is composed of dipole (about 25\%) and octupole (about 75\%). We note that the octupole component is significantly large in comparison with the $4 \mathrm{u}$ states of $\mathrm{UO}_{2}$ and $\mathrm{NpO}_{2}$. In any case, as we have expected, we find only magnetic $4 \mathrm{u}$ moment originating from $\Gamma_{7}^{-}$ground state for $\mathrm{AmO}_{2}$.

Then, we increase the value of $r$ in order to move to the side of the $L S$ coupling scheme. In Fig. 4(b), we plot $T \chi_{\gamma}$ of the multipole susceptibility for $n=5$ and $r=1.5$ with the $\Gamma_{8}^{-}$ground state (see Fig. 2). In this case, we find that the primary component is $3 \mathrm{~g}$, which is mainly composed of quadrupole. The secondary component is $4 \mathrm{u}$ and we also find $5 \mathrm{~g}, 4 \mathrm{u}$, and $5 \mathrm{u}$ components with smaller eigenvalues. We note that some multipoles of $\mathrm{AmO}_{2}$ are the same as those in $\mathrm{NpO}_{2}$, except for $2 \mathrm{u}$ and $5 \mathrm{~g}$, although the corresponding eigenvalues are different.

Now we provide a comment on the value of $r$, which is introduced so as to increase the effect of Coulomb interactions and decrease the magnitude of spin-orbit coupling. Note, however, that the angular momentum coupling of the $5 f$ states of Am is nowhere near as close to the $L S$ coupling limit as for Cm. ${ }^{31,33}$ Thus, we should not entirely or strongly suppress the spin-orbit coupling in the $5 f$ states of Am. It is acceptable that we change slightly spin-orbit coupling and Coulomb interaction. Accordingly, when the value of $r$ is increased so as to move towards the side of the $L S$ coupling scheme, we should pay due attention not to suppress spin-orbit coupling too much.

\section{DISCUSSION AND SUMMARY}

In this paper, we have discussed the multipole state in the low-temperature region, by analyzing the sevenorbital impurity Anderson model with the use of the NRG method. We have found the multipole state for $n=2,3$, and 4 , which are not in contradiction to the phases observed in $\mathrm{UO}_{2}, \mathrm{NpO}_{2}$, and $\mathrm{PuO}_{2}$, respectively. Note here that we determine the candidates which will order in the actual periodic system at low temperatures.

For the case of $n=5$, when we use the same parameters as those for $n=2 \sim 4$, we have suggested the phase dominated by magnetic moment. However, if we change slightly Coulomb interactions and spin-orbit coupling, we have found the $\Gamma_{8}^{-}$quartet ground state for $\mathrm{AmO}_{2}$. In this situation, we have shown that the low-temperature phase can contain multipoles such as quadrupole and octupole. The parameters are artificially introduced here, but our purpose is to point out a possibility of the $\Gamma_{8}^{-}$ quartet ground state due to the competition between Coulomb interactions and spin-orbit coupling.

Unfortunately, we cannot determine the kind of multipole order only from the present calculation, but on the basis of the same crystal structure, it is plausible that $5 \mathrm{u}$ octupole order also appears in $\mathrm{AmO}_{2}$. On the other hand, it may be possible to exploit other scenar- 
ios, e.g., quadrupole ordering, which were invented for understanding of $\mathrm{NpO}_{2}$. In any cases, the combination of phenomenological theory and microscopic experiment will be useful to finalize the kind of multipole which orders at low temperatures in $\mathrm{AmO}_{2}$.

Experimentally it has been considered that the $\mathrm{CEF}$ ground state of $\mathrm{AmO}_{2}$ is $\Gamma_{7}^{-}$ground state. However, we believe that it is still meaningful to examine the experimental results on the basis of the $\Gamma_{8}^{-}$quartet ground state, although it may be difficult to perform the microscopic experiments of $\mathrm{AmO}_{2}$.

Finally, let us provide a comment on the simplification of the model. In this paper, since we have considered only single conduction band, there exists residual entropy in the results. In actuality, it should be finally released when we consider $t_{1 \mathrm{u}}$ and $t_{2 \mathrm{u}}$ conduction bands. This point is also related to the relevant multipole moment when we consider the ordered state in the periodic systems. In this sense, the present results are qualitative, but they include the actual multipole which forms ordered state.

In summary, we have discussed the multipole state of actinide dioxides due to the evaluation of the multipole susceptibility of the Anderson model. When Coulomb interaction and spin-orbit coupling have been appropriately changed, it has been found that multipoles including quadrupole and octupole are relevant to $\mathrm{AmO}_{2}$. It is believed that multipole ordering can be detected in $\mathrm{AmO}_{2}$ in future experiments.

\section{Acknowledgments}

The author is grateful to S. Kambe and Y. Tokunaga for fruitful discussions and useful comments. This work has been supported by a Grant-in-Aid for for Scientific Research on Innovative Areas "Heavy Electrons" (No. 20102008) of The Ministry of Education, Culture, Sports, Science, and Technology, Japan. The computation in this work has been done using the facilities of the $\mathrm{Su}$ percomputer Center of Institute for Solid State Physics, University of Tokyo.
1 P. Santini, R. Lémanski, and P. Erdös, Adv. Phys. 48, 537 (1999).

2 P. Santini, S. Carretta, G. Amoretti, R. Caciuffo, N. Magnani, and G. H. Lander, Rev. Mod. Phys. 81, 807 (2009).

3 R. Caciuffo, G. Amoretti, P. Santini, G. H. Lander, J. Kulda, and P. de V. Du Plessis, Phys. Rev. B 59, 13892 (1999).

4 L. E. Roy, T. Durakiewicz, R. L. Martin, J. E. Peralta, G. E. Scuseria, C. G. Olson, J. J. Joyce, and E. Guziewicz, J. Comp. Chem. 29, 2288 (2008).

${ }^{5}$ G. Amoretti, A. Blaise, R. Caciuffo, J. M. Fournier, M. T. Hutchings, R. Osborn, and A. D. Taylor, Phys. Rev. B 40, 1856 (1989).

${ }^{6}$ E. F. Westrum, Jr., J. B. Hatcher, and D. W. Osborne, J. Chem. Phys. 21, 419 (1953).

7 J. W. Ross and D. J. Lam, J. Appl. Phys. 38, 1451 (1967).

${ }^{8}$ P. Erdös G. Solt, Z. Zolnierek, A. Blaise, and J. M. Fournier, Physica 102B, 164 (1980).

9 P. Santini and G. Amoretti, Phys. Rev. Lett. 85, 2188 (2000).

10 J. A. Paixão, C. Detlefs, M. J. Longfield, R. Caciuffo, P. Santini, N. Bernhoeft, J. Rebizant, and G. H. Lander, Phys. Rev. Lett. 89, 187202 (2002).

11 R. Caciuffo, J. A. Paixão, C. Detlefs, M. J. Longfield, P. Santini, N. Bernhoeft, J. Rebizant, and G. H. Lander, J. Phys.: Condens. Matter 15, S2287 (2003).

12 S. W. Lovesey, E. Balcar, C. Detlefs, G. van der Laan, D. S. Sivia, and U. Staub, J. Phys.: Condens. Matter 15, 4511 (2003).

13 A. Kiss and P. Fazekas, Phys. Rev. B 68, 174425 (2003).

14 J. M. Fournier, A. Blaise, G. Amoretti, R. Caciuffo, J. Larroque, M. T. Hutchings, R. Osborn, and A. D. Taylor, Phys. Rev. B 43, 1142 (1991).

15 Y. Tokunaga, Y. Homma, S. Kambe, D. Aoki, H. Sakai, E. Yamamoto, A. Nakamura, Y. Shiokawa, R. E. Walstedt, and H. Yasuoka, Phys. Rev. Lett. 94, 137209 (2005).

16 N. Magnani, S. Carretta, R. Caciuffo, P. Santini, G. Amoretti, A. Hiess, J. Rebizant, and G. H. Lander, Phys. Rev. B 78, 104425 (2008).

17 K. Kubo and T. Hotta, Phys. Rev. B 71, 140404(R) (2005).

18 K. Kubo and T. Hotta, Phys. Rev. B 72, 144401 (2005).

19 S. Kern, C.-K. Loong, G. L. Goodman, B. Cort, and G. H. Lander, J. Phys.: Condens. Matter 2, 1933 (1990).

20 S. Kern, R. A. Robinson, H. Nakotte, G. H. Lander, B. Cort, P. Watson, and F. A. Vigil, Phys. Rev. B 59, 104 (1999).

21 G. M. Kalvius, S. L. Ruby, B. D. Dunlap, G. K. Shenoy, D. Cohen, and M. B. Brodsky, Phys. Lett. 29B, 489 (1969),

22 D. G. Karraker, J. Phys. Chem. 63, 3174 (1975).

23 A. Bøuf, J. M. Fournier, J. F. Gueugnon, L. Manes, J. Rebizant, and F. Rustichelli, J. de Physique 40, L335 (1979).

24 M. M. Abraham, L. A. Boatner, C. B. Finch, and R. W. Reynolds, Phys. Rev. B 3, 2864 (1971).

${ }^{25}$ W. Kolbe, N. Edelstein, C. B. Finch, and M. M. Abraham, J. Chem. Phys. 60, 607 (1974).

26 N. Magnani, P. Santini, G. Amoretti, and R. Caciuffo, Phys. Rev. B 71, 054405 (2005).

27 T. Hotta and H. Harima, J. Phys. Soc. Jpn. 75, 124711 (2006).

28 T. Hotta, J. Phys. Soc. Jpn. 76, 034713 (2007).

${ }^{29}$ K. T. Moore, G. van der Laan, R. G. Haire, M. A. Wall, and A. J. Schwartz, Phys. Rev. B 73, 033109 (2006).

${ }^{30}$ K. T. Moore, G. van der Laan, M. A. Wall, A. J. Schwartz, and R. G. Haire, Phys. Rev. B 76, 073105 (2007).

31 K. T. Moore, G. van der Laan, R. G. Haire, M. A. Wall, A. J. Schwartz, and P. Soderlind, Phys. Rev. Lett. 98, 236402 (2007).

32 K. T. Moore and G. van der Laan, Rev. Mod. Phys. 81, 235 (2009).

33 J. H. Shim, K. Haule, and G. Kotliar, Europhys. Lett. 
$\mathbf{8 5}, 17007$ (2009)

34 J. C. Slater, Phys. Rev. 34, 1293 (1929).

35 E. U. Condon and G. H. Shortley, Phys. Rev. 37, 1025 (1931).

36 J. A. Gaunt, Phil. Trans. Roy. Soc. A228, 195 (1929).

37 G. Racah, Phys. Rev. 62, 438 (1942).

38 M. T. Hutchings, Solid State Phys. 16, 227 (1964).

${ }^{39}$ K. R. Lea, M. J. M. Leask, and W. P. Wolf, J. Phys. Chem. Solids 23, 1381 (1962).

40 E. Eliav, U. Kaldor, and Y. Ishikawa, Phys. Rev. A 51, 225 (1995).

41 K. W. H. Stevens, Proc. Phys. Soc. A65, 209 (1952).
42 T. Maehira and T. Hotta, J. Magn. Magn. Mater. 310, 754 (2007).

43 T. Hotta, J. Phys. Soc. Jpn. 74, 2425 (2005).

44 T. Hotta, J. Phys. Soc. Jpn. 76, 083705 (2007).

45 T. Hotta, J. of Alloys Compd. 444-445, 162 (2007).

46 T. Hotta, J. Magn. Magn. Mater. 310, 1691 (2007).

47 T. Hotta, J. Phys. Soc. Jpn. 77, 074716 (2008).

48 T. Hotta, J. Phys. Soc. Jpn. 77 Suppl. A, 968 (2008).

49 H. R. Krishna-murthy, J. W. Wilkins, and K. G. Wilson, Phys. Rev. B 21, 1003 (1980). 